EPJ manuscript No.

(will be inserted by the editor)

\title{
Polaron cross-overs and $d$-wave superconductivity in Hubbard-Holstein model
}

R. Ramakumar and A. N. Das

Condensed Matter Physics Group, Saha Institute of Nuclear Physics, 1/AF Bidhannagar, Calcutta-700064, INDIA

Received: 26 May 2004 / Revised version: 9 July 2004

\begin{abstract}
We present a theoretical study of superconductivity of polarons in the Hubbard-Holstein model. A residual kinematic interaction proportional to the square of the polaron hopping energy between polarons and phonons provides a pairing field for the polarons. We find that superconducting instability in the $d$ wave channel is possible with small transition temperatures which is maximum in the large to small polaron cross-over region. An $s$-wave instability is found to be not possible when the effective on-site interaction between polarons is repulsive.
\end{abstract}

PACS. 63.20.Kr Phonon-electron and Phonon-Phonon interactions - 74.20.Mn Polarons and bipolarons

- 74.20.Fg BCS theoy and its development

\section{Introduction}

Polaronic superconductivity has been a subject of interest following discovery of superconductivity in cuprate oxides and some molecular conductors [1]. A characteristic feature of these materials is that the conduction electrons live in a narrow energy band. It is also recognized that electron-phonon $(e-p h)$ interaction is considerable in these materials. The simplest model for narrow band electrons

a Present Address: Department of Physics and Astrophysics, University of Delhi, Delhi-11000\%, India. interacting with local phonons is the Hubbard-Holstein model 2,3 . If the $e-p h$ interaction is large enough, it can lead to the formation of polarons. Building on an earlier work 4, Takada and Hotta recently studied [5] this model in the strong e-ph coupling limit where the polarons are small polarons. For intermediate $e-p h$ coupling, however, the polarons may not be small polarons depending on $e$ $p h$ coupling strength and the ratio of the values of the electronic hopping to the phonon energy. In this paper we present a study of superconductivity in this range to check 
the role of large-to-small polaron crossover on superconductivity.

\section{Mean-field theory of superconductivity in the Hubbard-Holstein model}

The Hubbard-Holstein (HH) model [2,3] is:

$$
\begin{aligned}
H & =-t \sum_{i \delta \sigma} c_{i \sigma}^{\dagger} c_{i+\delta \sigma}+U \sum_{i} n_{i \uparrow} n_{i \downarrow} \\
& +\sum_{i} b_{i}^{\dagger} b_{i}+g \sum_{i \sigma} n_{i \sigma}\left(b_{i}^{\dagger}+b_{i}\right) .
\end{aligned}
$$

Here $t(>0)$ is the hopping energy between molecules at lattice site $i$ and its nearest-neighbor lattice sites $\boldsymbol{i}+\boldsymbol{\delta}$, $c_{i \sigma}\left(c_{i \sigma}^{\dagger}\right)$ is the annihilation (creation) operator for the electron with spin $\sigma$ at a lattice site $i$ and $n_{i \sigma}$ is the corresponding number operator, $U$ is on-site Coulomb repulsion, $b_{i}\left(b_{i}^{\dagger}\right)$ is the phonon annihilation (creation) operator, and $g$ is the $e$-ph interaction strength. All energies are measured in terms of the phonon energy $(\hbar \omega)$. In the calculations given below, first we apply a Modified-LangFirsov (MLF) transformation [6] (which is the original LF [7] transformation modified to include lattice deformations on the sites nearest neighbor to the site where an electron resides) to $H$ to convert it to the polaron representation, then we eliminate the residual polaron-phonon (pol-ph) interactions to obtain an effective interaction between polarons, and finally we do a mean-field theory of superconductivity specializing to the cases of $s$ and $d$ wave order parameters in the case of a square lattice. Our main result is contained in Fig. 2, where it is shown that the superconducting transition temperature in the $d$-wave channel goes through its maximum in the large to small polaron crossover region (see Fig. 1). The details of our calculations are given in the rest of this section and the conclusions are given in Sec. III.

The application of MLF transformation to $H$ leads to $H_{M L F}=e^{R} H e^{-R}$, where $R$ is given by,

$$
R=\lambda_{\circ} \sum_{i \sigma} n_{i \sigma}\left(b_{i}^{\dagger}-b_{i}\right)+\lambda_{1} \sum_{i \delta \sigma} n_{i \sigma}\left(b_{i+\delta}^{\dagger}-b_{i+\delta}\right),
$$

$\lambda_{0}$ and $\lambda_{1}$ represent the lattice deformations at the electron site and its nearest-neighbor sites, respectively. They are treated as variational parameters to be determined by minimization (with respect to $\lambda_{\circ}$ and $\lambda_{1}$ ) of ground state energy of the transformed Hamiltonian. Using the above $R$, we obtain:

$$
e^{R} c_{i \sigma} e^{-R}=c_{i \sigma} \exp \left[-\lambda_{\circ}\left(b_{i}^{\dagger}-b_{i}\right)-\lambda_{1} \sum_{\delta}\left(b_{i+\delta}^{\dagger}-b_{i+\delta}\right)\right]
$$

and,

$$
e^{R} b_{i} e^{-R}=b_{i}-\lambda_{\circ} \sum_{\sigma} n_{i \sigma}-\lambda_{1} \sum_{\delta \sigma} n_{i+\delta, \sigma} .
$$

The transformed Hamiltonian $\left(H_{M L F}\right)$ is obtained as:

$$
H_{M L F}=H_{\circ}+H_{1}+H_{2},
$$

where,

$$
\begin{aligned}
H_{\circ} & =\sum_{i} b_{i}^{\dagger} b_{i}-\epsilon_{p} \sum_{i \sigma} n_{i \sigma}+U_{e f f} \sum_{i} n_{i \uparrow} n_{i \downarrow} \\
& -V_{1} \sum_{i \delta} \sum_{\sigma \sigma^{\prime}} n_{i \sigma} n_{i+\delta, \sigma^{\prime}} \\
& +V_{2} \sum_{i \delta \delta^{\prime}, \delta \neq \delta^{\prime}} \sum_{\sigma \sigma^{\prime}} n_{i+\delta, \sigma} n_{i+\delta^{\prime}, \sigma^{\prime}}, \\
H_{1} & =-t \sum_{i \delta \sigma} c_{i \sigma}^{\dagger} c_{i+\delta, \sigma} \exp \left(X_{i}-X_{i+\delta}\right),
\end{aligned}
$$

and

$$
H_{2}=\left(g-\lambda_{\circ}\right) \sum_{i \sigma} n_{i \sigma}\left(b_{i}^{\dagger}+b_{i}\right)
$$


R. Ramakumar, A. N. Das: Polaron cross-overs and $d$-wave superconductivity in Hubbard-Holstein model

$$
-\lambda_{1} \sum_{i \delta \sigma} n_{i \sigma}\left(b_{i+\delta}^{\dagger}+b_{i+\delta}\right)
$$

Here,

$$
\begin{gathered}
\epsilon_{p}=\lambda_{\circ}\left(2 g-\lambda_{\circ}\right)-z \lambda_{1}^{2}, \\
U_{e f f}=U-2 \epsilon_{p}, \\
V_{1}=2\left(g-\lambda_{\circ}\right) \lambda_{1}, V_{2}=\lambda_{1}^{2}, \\
X_{i}=\lambda_{\circ}\left(b_{i}^{\dagger}-b_{i}\right)+\lambda_{1} \sum_{\delta}\left(b_{i+\delta}^{\dagger}-b_{i+\delta}\right),
\end{gathered}
$$

and $z$ is the coordination number of a lattice site. The Eq. (5) is the Hamiltonian of polarons. The polaron is much more massive than the original electron since it is a composite particle consisting of an electron and the associated lattice deformations. These polarons interact among themselves (through the interactions $U_{e f f}, V_{1}$ and $V_{2}$ ), and interact with the phonons through off-diagonal phonon terms of $H_{1}$ and $H_{2}$. The phonons remain unaffected by the MLF transformation. Notice also that the pol-ph couplings are much weaker than the original $e-p h$ coupling $(g)$.

Now we work using the variational phonon basis obtained through the MLF transformation. The variational parameters $\lambda_{0}$ and $\lambda_{1}$ are found out from a minimization of the ground-state energy in the normal state with zero phonon averaging. The values of $\lambda_{0}$ and $\lambda_{1}$ depend on $t / \omega$ and $g / \omega$ for a particular lattice and not on $U$. With this variational basis we proceed with our second order perturbation calculation by expanding the exponentials in $H_{1}$ to obtain, up to $O\left(\lambda_{\circ}-\lambda_{1}\right), \widetilde{H}$ valid for $\left(\lambda_{\circ}-\lambda_{1}\right)<1$ as:

$$
\widetilde{H}=H_{\circ}-t_{p} \sum_{i \delta \sigma} c_{i \sigma}^{\dagger} c_{i+\delta, \sigma}+H_{2}+H_{3}
$$

where,

$$
\begin{aligned}
H_{3} & =-t_{p} \sum_{i \delta \sigma} c_{i \sigma}^{\dagger} c_{i+\delta, \sigma}\left[\left(\lambda_{\circ}-\lambda_{1}\right)\left(b_{i}^{\dagger}-b_{i}-b_{i+\delta}^{\dagger}+b_{i+\delta}\right)\right. \\
& +\lambda_{1} \sum_{\delta^{\prime} \neq \delta}\left(b_{i+\delta^{\prime}}^{\dagger}-b_{i+\delta^{\prime}}\right) \\
& \left.-\lambda_{1} \sum_{\delta^{\prime} \neq-\delta}\left(b_{i+\delta+\delta^{\prime}}^{\dagger}-b_{i+\delta+\delta^{\prime}}\right)\right]
\end{aligned}
$$

and,

$$
t_{p}=t \exp \left[-\left(\lambda_{\circ}-\lambda_{1}\right)^{2}-(z-1) \lambda_{1}^{2}\right]
$$

In the above equation $H_{2}$ and $H_{3}$ describe the pol-ph interactions. On eliminating the pol-ph interaction terms and considering second order processes only, we obtain (for $\left.z t_{p}<1\right)$

$$
\widetilde{\widetilde{H}}=H_{\circ}-t_{p} \sum_{i \delta \sigma} c_{i \sigma}^{\dagger} c_{i+\delta, \sigma}+H_{4}
$$

where,

$$
\begin{aligned}
H_{4} & =-2\left(g-\lambda_{\circ}\right)^{2} \sum_{i} n_{i \uparrow} n_{i \downarrow} \\
& +2 \lambda_{1}\left(g-\lambda_{\circ}\right) \sum_{i \delta} \sum_{\sigma \sigma^{\prime}} n_{i \sigma} n_{i+\delta, \sigma^{\prime}} \\
& -\lambda_{1}^{2} \sum_{i \delta \delta^{\prime}} \sum_{\sigma \sigma^{\prime}} n_{i+\delta, \sigma} n_{i+\delta^{\prime}, \sigma^{\prime}} \\
& -t_{p}^{2}\left(\lambda_{\circ}-\lambda_{1}\right)^{2} \sum_{i \delta \delta^{\prime}} \sum_{\sigma \sigma^{\prime}}\left[c_{i-\delta^{\prime}, \sigma^{\prime}}^{\dagger} c_{i \sigma}^{\dagger} c_{i+\delta, \sigma} c_{i, \sigma^{\prime}}\right. \\
& +c_{i, \sigma^{\prime}}^{\dagger} c_{i-\delta, \sigma}^{\dagger} c_{i, \sigma} c_{i+\delta^{\prime}, \sigma^{\prime}}-c_{i-\delta^{\prime}, \sigma^{\prime}}^{\dagger} c_{i-\delta, \sigma^{\prime}}^{\dagger} c_{i \sigma} c_{i \sigma^{\prime}} \\
& \left.-c_{i \sigma^{\prime}}^{\dagger} c_{i \sigma}^{\dagger} c_{i+\delta, \sigma} c_{i+\delta^{\prime}, \sigma^{\prime}}\right] .
\end{aligned}
$$

It may be noted that our study is restricted to the region where $t_{p}\left(\lambda_{\circ}-\lambda_{1}\right),\left(g-\lambda_{\circ}\right)$, and $\lambda_{1}$ are much less than 1 even though $g>1$. This justifies our perturbation calculation. On simplification, confining to nearest neighbor singlet pairing terms, and neglecting terms $O\left(\lambda_{1}^{2}\right)$ since $\lambda_{1}$ is quite small (see Fig. 2), we get:

$$
\widetilde{\widetilde{H}}=-t_{p} \sum_{i \delta \sigma} c_{i \sigma}^{\dagger} c_{i+\delta, \sigma}-\epsilon_{p} \sum_{i \sigma} n_{i \sigma}+\widetilde{U} \sum_{i} n_{i \uparrow} n_{i \downarrow}
$$


R. Ramakumar, A. N. Das: Polaron cross-overs and $d$-wave superconductivity in Hubbard-Holstein model

$$
\begin{aligned}
& -t_{p}^{2}\left(\lambda_{\circ}^{2}-2 \lambda_{\circ} \lambda_{1}\right) \sum_{i \delta} \sum_{\sigma \sigma^{\prime}}\left[c_{i+\delta, \sigma^{\prime}}^{\dagger} c_{i, \sigma}^{\dagger} c_{i+\delta, \sigma} c_{i \sigma^{\prime}}\right. \\
& +c_{i+\delta, \sigma^{\prime}}^{\dagger} c_{i \sigma}^{\dagger} c_{i+\delta, \sigma} c_{i \sigma^{\prime}}-c_{i+\delta, \sigma^{\prime}}^{\dagger} c_{i+\delta, \sigma}^{\dagger} c_{i \sigma} c_{i \sigma^{\prime}} \\
& \left.-c_{i \sigma^{\prime}}^{\dagger} c_{i \sigma}^{\dagger} c_{i+\delta, \sigma} c_{i+\delta, \sigma^{\prime}}\right]
\end{aligned}
$$

$$
\begin{gathered}
\Delta_{\delta}=<c_{i+\delta \uparrow}^{\dagger} c_{i \downarrow}^{\dagger}>, \\
\widetilde{\widetilde{U}}=\widetilde{U}-z V_{t p},
\end{gathered}
$$

where $\widetilde{U}=\left(U-2 g^{2}\right)$. The elimination of the pol-ph terms generates a pairing field whose magnitude is $O\left(t_{p}^{2}\right)$ which is rather small. Note that the field associated with the onsite pair-hopping processes (the last two terms in $\widetilde{\widetilde{H}}$ ) is repulsive. The origin of superconductivity in this model, when $\tilde{U}>0$, then is the field generated through a virtual emission and absorption of phonons while the polarons hop from site to site. The two-polaron process involved is one polaron hops to its nearest neighbor site and excites a phonon at the target site and another polaron on the target site absorbs the excited phonon and hops to the site left by the first polaron. These processes lead to the fourth and fifth terms in If the $e$-ph interaction is not sufficient enough to lead to the formation of polarons, superconductivity occurs in the usual way through the phonon exchange between electrons, and the pairing field contains the full $e-p h$ interaction. Now we are at a stage where we can do a mean-field theory of the superconductivity using $\widetilde{\widetilde{H}}$.

On mean-field factorization of $\widetilde{\widetilde{H}}$, we obtain:

$$
\begin{aligned}
H_{M F} & =-t_{p} \sum_{i \delta \sigma} c_{i \sigma}^{\dagger} c_{i+\delta, \sigma}-\left(\epsilon_{p}-\widetilde{U} \frac{n}{2}\right) \sum_{i \sigma} n_{i \sigma}-N \widetilde{U} \frac{n^{2}}{4} \\
& +\widetilde{\widetilde{U}} \sum_{i}\left(A_{\circ} c_{i \downarrow} c_{i \uparrow}+\text { h.c. }\right)-N \widetilde{\widetilde{U}}\left|A_{\circ}\right|^{2} \\
& +V_{t p} \sum_{i \delta}\left(\Delta_{\delta} c_{i+\delta \downarrow} c_{i \uparrow}+\text { h.c. }\right)-N z V_{t p}\left|\Delta_{\delta}\right|^{2}
\end{aligned}
$$

where

$$
A_{\circ}=<c_{i \uparrow}^{\dagger} c_{i \downarrow}^{\dagger}>
$$

and

$$
V_{t p}=-4 t_{p}^{2}\left(\lambda_{\circ}^{2}-2 \lambda_{\circ} \lambda_{1}\right)
$$

The above mean-field approximation is valid for $\tilde{U}$ less than the polaron band-width. For larger $\tilde{U}$ strong correlation effects like Hubbard sub-band formation can occur. For recent studies of strong correlation effects originating from large $U$ in Hubbard-Holstein model see Refs. [8,9]. Converting to momentum space, we get:

$$
\begin{aligned}
H_{M F} & =-t_{p} \sum_{\mathbf{k} \sigma} \xi(\mathbf{k}) c_{\mathbf{k} \sigma}^{\dagger} c_{\mathbf{k} \sigma}-\left(\epsilon_{p}-\frac{\widetilde{U} n}{2}\right) \sum_{\mathbf{k} \sigma} n_{\mathbf{k} \sigma} \\
& +\sum_{\mathbf{k}}\left[\left(\widetilde{\widetilde{U}} A_{\circ}+V_{t p} A_{1}(\mathbf{k})\right) c_{-\mathbf{k} \downarrow} c_{\mathbf{k} \uparrow}+h . c .\right] \\
& -N \widetilde{\widetilde{U}}\left|A_{\circ}\right|^{2}-N z V_{t p}\left|\Delta_{\delta}\right|^{2},
\end{aligned}
$$

where

$$
\begin{gathered}
\xi(\mathbf{k})=\sum_{\delta} e^{i \mathbf{k} \cdot \delta}, \\
A_{1}(\mathbf{k})=\sum_{\delta} \Delta_{\delta} e^{i \mathbf{k} \cdot \delta},
\end{gathered}
$$

and $N$ is the number of lattice sites. In the $s$-wave case, $\widetilde{U}$ term should be considered even if $\widetilde{U}>0$ since the extended $s$-wave order parameter has an on-site component, and this is suppressed by the on-site repulsion $\widetilde{U}$. The $\widetilde{U}$ term does not affect $d$-wave pairing. In studying superconductivity, we will be considering only $\widetilde{U}>0$ case since $\widetilde{U}<0$ case leads to an effective attractive Hubbard model situation and this model is well understood $[10,11$, 12. Now we will consider $s$ and $d$ wave pairing channels separately. 


\section{1 s-wave channel}

In the $s$-wave case, the effective Hamiltonian is

$$
H_{s}=\sum_{\mathbf{k} \sigma} \tilde{\epsilon}(\mathbf{k}) c_{\mathbf{k} \sigma}^{\dagger} c_{\mathbf{k} \sigma}+\sum_{\mathbf{k}}\left(D_{s}(\mathbf{k}) c_{-\mathbf{k} \downarrow} c_{\mathbf{k} \uparrow}+\text { h.c. }\right)+s_{\circ}
$$

where, $\tilde{\epsilon}(\mathbf{k})=-t_{p} \xi(\mathbf{k})-\tilde{\mu}, D_{s}(\mathbf{k})=\widetilde{\widetilde{U}} A_{\circ}+V_{t p} A_{1}^{s}(\mathbf{k})$, $A_{1}^{s}(\mathbf{k})=2 \Delta_{\delta} \gamma_{s}(\mathbf{k}), \tilde{\mu}=\mu+\epsilon_{p}-\widetilde{U} n / 2, \gamma_{s}(k)=\cos \left(k_{x} a\right)+$ $\cos \left(k_{y} a\right)$ for a square lattice of lattice constant $a$, and $s_{\circ}=-\left(N \widetilde{U} n^{2} / 4\right)-n \widetilde{\widetilde{U}}\left|A_{\circ}\right|^{2}-N z V_{t p}\left|\Delta_{\delta}\right|^{2}$. In the above we have introduced a chemical potential $(\mu)$ to fix the number density, and $A_{1}^{s}(k)$ is for the isotropic case. The integral equation which determines $D_{s}(\mathbf{k})$ is obtained, using Green's functions, to be,

$$
D_{s}(\mathbf{k})=-\frac{1}{N} \sum_{\mathbf{k}^{\prime}} V_{s}\left(\mathbf{k}^{\prime}\right) \frac{D_{s}\left(\mathbf{k}^{\prime}\right)}{2 E_{s}\left(\mathbf{k}^{\prime}\right)} \tanh \left[\frac{\beta E_{s}\left(\mathbf{k}^{\prime}\right)}{2}\right],
$$

where the pairing field $V_{s}\left(\mathbf{k ~ k}^{\prime}\right)$ is

$$
V_{s}\left(\mathbf{k} \mathbf{k}^{\prime}\right)=\widetilde{\widetilde{U}}+4 z^{-1} V_{t p} \gamma_{s}(\mathbf{k}) \gamma_{s}\left(\mathbf{k}^{\prime}\right),
$$

$\beta=1 / k_{B} T, k_{B}$ is the Boltzmann constant, and the quasiparticle energy $E_{s}(\mathbf{k})=\sqrt{\tilde{\epsilon}^{2}(\mathbf{k})+\left|D_{s}(\mathbf{k})\right|^{2}}$. Now, we have already mentioned that we will consider the non-trivial case of $\widetilde{U}>0$. In that case $s$-wave pairing is not possible since $V_{s}\left(\mathbf{k} \mathbf{k}^{\prime}\right)$ is not attractive.

\section{$2.2 \mathrm{~d}$-wave channel}

In this case, we have

$$
H_{d}=\sum_{\mathbf{k} \sigma} \tilde{\epsilon}(\mathbf{k}) c_{\mathbf{k} \sigma}^{\dagger} c_{\mathbf{k} \sigma}+\sum_{\mathbf{k}}\left(D_{d}(\mathbf{k}) c_{-\mathbf{k} \downarrow} c_{\mathbf{k} \uparrow}+h . c .\right)+d_{\circ}
$$

where $D_{d}(\mathbf{k})=V_{t p} A_{1}(\mathbf{k})$, and $d_{\circ}=-\left(N U n^{2} / 4\right)-$ $N z V_{t p}\left|\Delta_{\delta}\right|^{2}$. Then the $d$-wave gap equation is obtained as

$$
D_{d}(\mathbf{k})=-\frac{1}{N} \sum_{\mathbf{k}^{\prime}} V_{d}\left(\mathbf{k} \mathbf{k}^{\prime}\right) \frac{D_{d}\left(\mathbf{k}^{\prime}\right)}{2 E_{d}\left(\mathbf{k}^{\prime}\right)} \tanh \left[\frac{\beta E_{d}\left(\mathbf{k}^{\prime}\right)}{2}\right],
$$

where,

$$
V_{d}\left(\mathbf{k} \mathbf{k}^{\prime}\right)=4 z^{-1} V_{t p} \gamma_{d}(\mathbf{k}) \gamma_{d}\left(\mathbf{k}^{\prime}\right)
$$

$E_{d}^{2}(\mathbf{k})=\tilde{\epsilon}^{2}(\mathbf{k})+\left|D_{d}(\mathbf{k})\right|^{2}$, and $\gamma_{d}(k)=\cos \left(k_{x} a\right)-\cos \left(k_{y} a\right)$ for a 2D-square-lattice case. The superconducting transition temperature $T_{c}^{d}$ is obtained from,

$$
1=-\frac{1}{N} \sum_{\mathbf{k}^{\prime}} V_{t p} \frac{\gamma_{d}^{2}\left(\mathbf{k}^{\prime}\right)}{2 \tilde{\epsilon}\left(\mathbf{k}^{\prime}\right)} \tanh \left[\frac{\beta_{c} \tilde{\epsilon}\left(\mathbf{k}^{\prime}\right)}{2}\right] .
$$

where $\beta_{c}=1 / k_{B} T_{c}^{d}$.

We have numerically analyzed the above equations and the results are shown in Figs. 1-2. The values of $t(<0.5)$ used are in the anti-adiabatic limit. In Fig. 1 we have displayed the variation of the lattice deformation $\left(\lambda_{\circ}\right.$ and $\lambda_{1}$ ) as a function of $g$. A smooth cross-over from a large to a small polaron [6] with increasing $g$ is seen in Fig. 1 .

The variation of $T_{c}$ with $g$ (for $n=0.81$ ) for superconducting instability in the $d$-wave channel is displayed in Fig. 2. The variation of $T_{c}$ with $n$ has a bell shaped form centered at half-filling similar to that found in Ref. [5]. Superconducting instability with small $T_{c}^{d}$ is possible for intermediate values of $e-p h$ coupling. The small $T_{c}^{d}$ is due to small value of the pairing field [which is $\left.O\left(t_{p}^{2}\right)\right]$. The $T_{c}^{d}$ is seen to reach a maximum in the large to small polaron cross-over region.

\section{Conclusions}

In this work we presented a study of superconductivity in the anti-adiabatic limit of the Hubbard-Holstein model 
considering the polaron formation. Our purpose was to study superconductivity of the polarons in the large to small polaron cross over region. We found that transition temperature for superconducting instability in the $d$-wave channel goes through its maximum in the large to small polaron cross-over regime. We also found that $s$-wave pairing is not possible for the case of $U>2 g^{2}$. In the strong $e-p h$ coupling limit also, it was found $[5]$ that $T_{c}^{d}$ is extremely small when $U>2 g^{2}$. In real materials there is inter-site Coulomb repulsion and this will drastically reduce [13] the $T_{c}^{d}$. So, we conclude that, when the mechanism considered here operates, $T_{c}^{d}$ is very small both in the intermediate and strong coupling limits of the $e-p h$ interaction.

\section{References}

1. A. S. Alexandrov and N. F. Mott, Rep. Prog. Phys. 57, 1197 (1994); Polarons and Bipolarons (World Scientific, Singapore, 1995).

2. J. Hubbard, Proc. of Roy. Soc. of London A 281, 401(1964).

3. T. Holstein, Ann. Phys. 8, 325(1959).

4. G. Beni, P. Pincus, and J. Kanamori, Phys. Rev. B 10, 1896(1974).

5. Y. Takada and T. Hotta, Int. J. Mod. Phys. B 12, $3042(1998)$.

6. A. N. Das and S. Sil, J. Phys. CM 5, 8265(1993).

7. I. G. Lang and Yu. A. Firsov, Z. Éksp. Teor. Fiz. 43, 1843(1962) [Sov. Phys. JETP 16, 1301(1962)].

8. M. Capone, G. Sangiovanni, C. Castellani, C. Di Castro, and M. Grilli, Phys. Rev. Lett. 92, 106401 (2004).

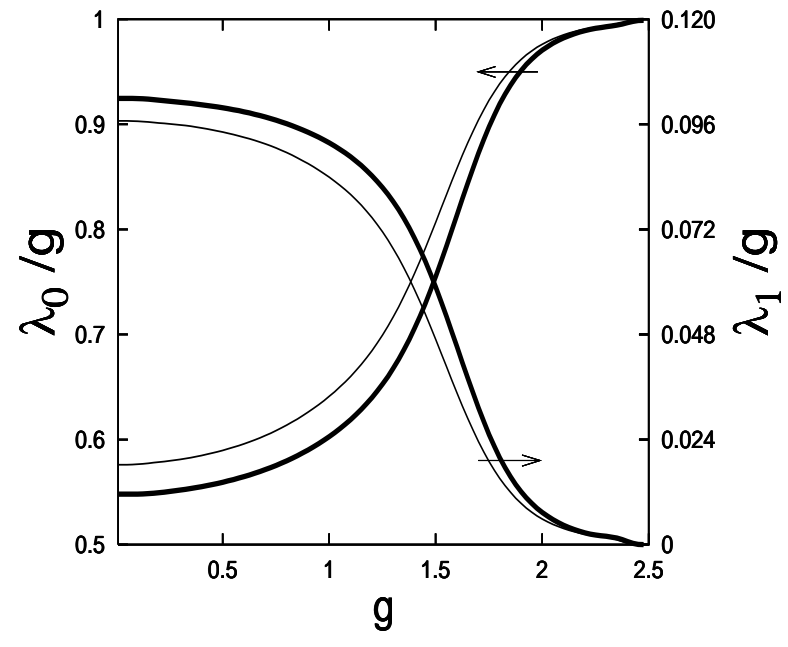

Fig. 1. The variational parameters $\lambda_{0}$ and $\lambda_{1}$ for $n=0.81$ and $t: 0.35$ (thick lines) and 0.30 (thin lines).

9. W. Koller, D. Meyer, Y. Ono, and A. C. Hewson, Europhys. Lett. 66, 559 (2004).

10. R. Micnas, J. Ranninger, and S. Robaszkiewicz, Rev. Mod. Phys. 62, 113(1990).

11. M. Keller, W. Metzner, and U. Schollwöck, Phys. Rev. Lett. 86, 4612(2001).

12. A. Sewer, X. Zotos, and H. Beck, Phys. Rev. B 66, $140504(2002)$

13. An intersite repulsion term $U_{1} \sum_{i \delta} \sum_{\sigma \sigma^{\prime}} n_{i+\delta, \sigma} n_{i, \sigma^{\prime}}$ reduces the pairing field by a factor of $2 U_{1}$. 
R. Ramakumar, A. N. Das: Polaron cross-overs and $d$-wave superconductivity in Hubbard-Holstein model

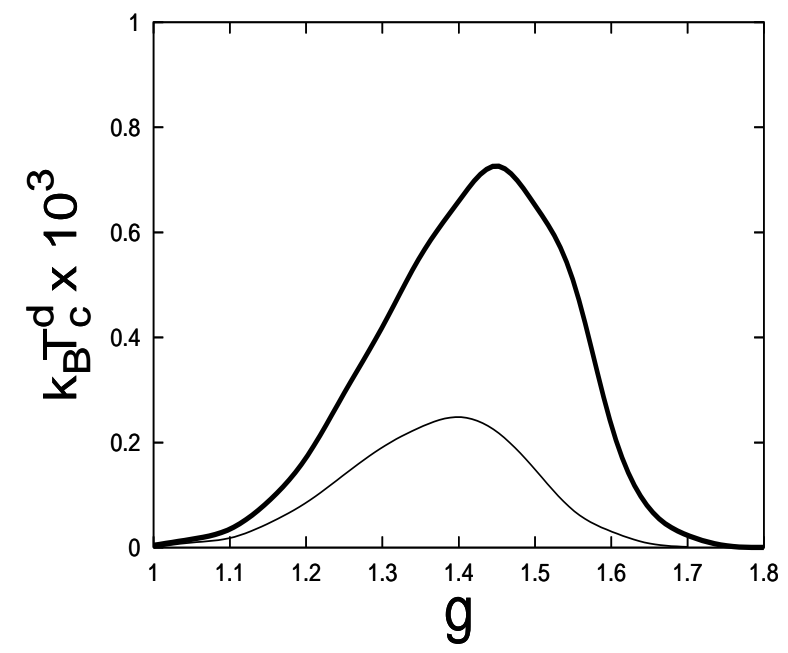

Fig. 2. $T_{c}^{d}$ vs $g$ for $n=0.81$ and $t$ : 0.35 (thick line) and 0.30 (thin line). 